INPLASY

PROTOCOL

To cite: Jia et al. The effects of ACEI/ARB, aldosterone receptor antagonists and statins on preventing recurrence of atrial fibrillation: a protocol for systematic review and network metaanalysis. Inplasy protocol 202090004. doi:

10.37766/inplasy2020.9.0004

Received: 01 September 2020

Published: 01 September 2020

Corresponding author:

Qiulei Jia

lotusrosa@163.com

Author Affiliation:

Department of cardiovascular, Guang'anmen Hospital, China Academy of Chinese Medical Sciences

Support: No funding for this work.

Review Stage at time of this submission: The review has not yet started.

Conflicts of interest:

The authors report no conflicts of interest.

\section{The effects of ACEI/ARB, aldosterone receptor antagonists and statins on preventing recurrence of atrial fibrillation: a protocol for systematic review and network meta-analysis}

Review question / Objective: P: Patients with atrial fibrillation treated with electrical cardioversion; I: Angiotensinconverting enzyme inhibitors (ACEI), angiotensin II receptor blockers (ARB), aldosterone receptor antagonists or statin combined with standard therapy, including rate control, anticoagulants and antiarrhythmic; C: placebo, or no drug combined with standard therapy; $O$ : The primary endpoint is the time to a first electrocardiographically confirmed recurrence of atrial fibrillation. The secondary endpoints are (1) Left atrial dimension in echocardiography; (2) adverse drug events/reactions (ADEs/ADRs).

Condition being studied: Atrial fibrillation is the most common cardiac arrhythmia. Restoration and long-term maintenance of sinus rhythm is challenging. Renin-angiotensin-aldosterone system (RAAS) modulators and statins have been used for the upstream therapy of AF. This study aims to evaluate the efficacy and safety of ACEI/ARB, aldosterone receptor antagonists and statin on preventing recurrence of atrial fibrillation.

INPLASY registration number: This protocol was registered with the International Platform of Registered Systematic Review and Meta-Analysis Protocols (INPLASY) on 01 September 2020 and was last updated on 01 September 2020 (registration number INPLASY202090004).

\section{INTRODUCTION}

Review question / Objective: P: Patients with atrial fibrillation treated with electrical cardioversion; I: Angiotensin-converting enzyme inhibitors (ACEI), angiotensin II receptor blockers (ARB), aldosterone receptor antagonists or statin combined 
with standard therapy, including rate control, anticoagulants and antiarrhythmic; C: placebo, or no drug combined with standard therapy; 0 : The primary endpoint is the time to a first electrocardiographically confirmed recurrence of atrial fibrillation. The secondary endpoints are (1) Left atrial dimension in echocardiography; (2) adverse drug events/reactions (ADEs/ADRs).

Condition being studied: Atrial fibrillation is the most common cardiac arrhythmia. Restoration and long-term maintenance of sinus rhythm is challenging. Reninangiotensin-aldosterone system (RAAS) modulators and statins have been used for the upstream therapy of AF. This study aims to evaluate the efficacy and safety of ACEI/ARB, aldosterone receptor antagonists and statin on preventing recurrence of atrial fibrillation.

\section{METHODS}

Participant or population: Adult patients diagnosed with AF treated with electrical cardioversion will be included. The diagnostic criteria for AF will be referred to 2016 European society of cardiology preliminary criteria for the clinical diagnosis of AF and the AHA/ACA/HRS 2019 guidelines for the management of patients with AF.

Intervention: Angiotensin-converting enzyme inhibitors (ACEI), angiotensin II receptor blockers (ARB), aldosterone receptor antagonists or statin combined with standard therapy, including rate control, anticoagulants and antiarrhythmic.

Comparator: Patients in the control group are those who have received placebo, or no drug combined with standard therapy.

Study designs to be included: Randomized controlled trials.

Eligibility criteria: Eligibility criteria: (1) Randomized controlled trials (RCTs); (2) Participants diagnosed as AF by the criteria listed in 2016 European society of cardiology preliminary criteria for the clinical diagnosis of AF and the AHA/ACA/ HRS 2019 guidelines for the management of patients with AF. (3) The intervention is Angiotensin-converting enzyme inhibitors (ACEI), angiotensin II receptor blockers (ARB), aldosterone receptor antagonists or statin combined with standard therapy, including rate control, anticoagulants and antiarrhythmic. Comparison intervention is placebo, or no drug combined with standard therapy; (4) Outcomes: The primary endpoint is the time to a first electrocardiographically confirmed recurrence of atrial fibrillation. The secondary endpoints are (a) Left atrial dimension in echocardiography; (b) adverse drug events/reactions (ADEs/ ADRs).

Information sources: We will search MEDLINE, EMBASE, The Cochrane Library, ClinicalTrials.gov, which are updated on Aug 31, 2020.

Main outcome(s): The primary endpoint is the time to a first electrocardiographically confirmed recurrence of atrial fibrillation.

Additional outcome(s): The secondary endpoints are (a) Left atrial dimension in echocardiography; (b) adverse drug events/ reactions (ADEs/ADRs).

Quality assessment / Risk of bias analysis: The methodological quality of trials will be assessed independently using criteria from the Cochrane Handbook for Systematic Review of Interventions, Version 5.1.0. Seven domains are considered such as sequence generation (selection bias), allocation concealment (selection bias), blinding of participants and personnel (performance bias), blinding of outcome assessment (detection bias), incomplete outcome data (attrition bias), selective outcome reporting (reporting bias), and other bias. Three levels of "low risk", "high risk", or "unclear risk" will be the quality appraisal category.

Strategy of data synthesis: We will conduct both standard meta-analyses of each pairwise direct comparison between interventions and a network meta-analysis 
combining results of all these comparisons in one analysis, exploiting both the direct comparisons within trials and the indirect comparisons across trials for each outcome. The pair-wise meta-analysis will be performed by STATA 14.0 software. The fixed-effects model will be used in pairwise meta-analysis and the summary statistics of odds ratio (OR) for dichotomous outcomes or standardized mean differences (SMD) with $95 \%$ Cls. Chisquare tests for heterogeneity will be used to assess between-study heterogeneity for each outcome, and the extent of the observed heterogeneity in effect sizes between studies will be assessed by the $\mathbf{I}$. The network meta-analysis will be implemented in a Bayesian framework using WinBUGS 1.4.3 (MRC Biostatistics Unit, Cambridge, UK). The Markov Chains Monte Carlo sampler will be used to generate samples. A total of $\mathbf{5 0 0 0}$ simulations for each chain will be set as the 'burn-in' period. Then, posterior summaries will be based on 100000 subsequent simulations. The results of the network meta-analysis will be reported as ORs or SMD with $95 \%$ Cls. To rank the treatments for each outcome, we will use the surface under the cumulative ranking curve (SUCRA) and the mean ranks. The network plots will be drawn using STATA 14.0 software. A comparison-adjusted funnel plot will be used to assess the publication bias using STATA 14.0 software.

Subgroup analysis: If the $12>50 \%$, we will explore sources of heterogeneity by subgroup analysis and meta-regression.

Sensibility analysis: Sensitivity analysis will be completed by changing the effect model or removing 1 study at a time to investigate the influence of a single study on the overall pooled estimate.

\section{Country(ies) involved: China.}

Keywords: ACEI; ARB; aldosterone receptor antagonists; statins; atrial fibrillation; network meta-analysis.
Author 2 - Wenbing Han. Author 3 - Shuqing Shi. Author 4 - Yuanhui Hu.

Contributions of each author:

Author 1 - Qiulei Jia. 